\title{
Advances and limitations of in vitro embryo production in sheep and goats
}

\author{
A. Menchaca ${ }^{1,3}$, N. Barrera ${ }^{1}$, P.C. dos Santos Neto ${ }^{1}$, F. Cuadro ${ }^{1}$, M. Crispo ${ }^{2}$ \\ ${ }^{1}$ Instituto de Reproducción Animal Uruguay, Fundación IRAUy, Montevideo, Uruguay. \\ ${ }^{2}$ Unidad de Animales Transgénicos y de Experimentación, Institut Pasteur de Montevideo, Uruguay.
}

\begin{abstract}
This review summarizes the latest advances and main limitations for the implementation of in vitro embryo production programs in sheep and goats. We describe the laparoscopic assisted technique for oocyte retrieval and propose new insights for follicular manipulation to improve oocyte quality. Further description of the routine conducted in our laboratory for the in vitro process of oocyte maturation, fertilization and embryo culture is presented, with emphasis in the main issues for the success of the technique. Protocols for fixed time embryo transfer (FTET) are proposed and the optimal number of in vitro produced (IVP) embryos to be transferred per female is discussed. In addition, we present pregnancy outcomes and birth rates recently obtained with FTET with IVP embryos cryopreserved by vitrification with new minimum volume methods. In summary, due to important refinements for in vitro embryo production in sheep and goats achieved in the recent years, this technology is now available for its implementation in commercial programs for genetic improvement, for the production of genetically engineered sheep and goats, and for basic research in reproduction.
\end{abstract}

Keywords: cryopreservation; ewe, goat, LOPU, superovulation.

\section{Follicular aspiration by laparoscopy}

The success of an in vitro embryo production (IVEP) program for either research purposes or industry application depends largely on the availability of a continuous number of good quality oocytes. Although slaughterhouse ovaries represent a very useful cheap and rich oocyte source for research projects, the application of this technology in the industry and livestock requires oocyte retrieval from live animals. Follicular aspiration by laparoscopy (laparoscopic ovum pick-up; LOPU) is the best available technique to obtain oocytes from sheep and goats, firstly described by Robin Tervit in New Zealand and Hernan Baldassarre in Argentina in the early 1990s (Tervit et al., 1992; Baldassarre et al., 1994). This approach has the advantage of eliminating the poor results observed with the traditional laparotomy technique, besides the possibility of repeating the procedure several times in the same female, and allowing the production of offspring from prepubertal animals (Baldassarre and Karatzas, 2004). LOPU is used to recover oocytes for conventional IVP, but also for the production of zygotes for the generation of transgenic founders (Baldassarre et al., 2003b) or for propagating transgenic animals by somatic cell nuclear transfer (Baldassarre et al., 2003a). Recently, this technique has also been proposed as a useful tool to assist the generation of genome edited sheep and goats (e.g. CRISPR system; Menchaca et al., 2016).

During the LOPU procedure, the animal is restrained on a standard laparoscopy table under general anesthesia. In our laboratory, general anesthesia is induced with ketamine and diazepam by i.v. route and is maintained with isofluorane inhalatory anesthesia. LOPU technique is further described by $\mathrm{H}$. Baldassarre (Menchaca et al., 2016), and briefly, consist of a laparoscopy equipment with a $5 \mathrm{~mm}$ diameter and $0^{\circ}$ angle telescope, two $5.5 \mathrm{~mm}$ trocar/cannula sets (one for the laparoscope and one for the forceps), one 3.5-5 mm trocar/cannula set for the aspiration pipette, a $5 \mathrm{~mm}$ atraumatic grasping forceps, a fiber optic cable and a light source. The oocyte aspiration set consists of a collection tube with an inlet connected through tubing to the aspiration pipette and an outlet connected through tubing to a vacuum pump. The aspiration pipette consists of a $20 \mathrm{G}$ short bevel needle glued to the tip of a $30 \mathrm{~cm}$-long acrylic tubing, with a $3 \mathrm{~mm}$ external diameter and $1 \mathrm{~mm}$ internal diameter. The ovarian surface is exposed by pulling from the fimbria in different directions using the forceps, and the follicles are punctured one by one using the aspiration pipette. For a trained team, in average it takes about 15-20 min per donor (including preparation) and recovery rate of cumulus-oocyte complex (COC) ranges from 50 to $90 \%$.

The possibility to use prepubertal females of 13 month old as oocyte source is another advantage of the LOPU technique, reducing the generational interval and accelerating the process of genetic improvement (Paramio, 2010). In addition, LOPU can be performed repeatedly in the same female with an interval between aspiration sessions of 4 days without affecting oocyte retrieval and quality (Gibbons et al., 2007). These two possibilities remarkably increases the capacity of multiplication of a female. The incidence of injuries caused to the ovary after successive LOPU sessions has been under study. In general, most of the reports did not found severe ovarian lesions that interfere with follicular aspiration, oocyte retrieval or embryo yield after 3 to 7 repeated hormonal treatments and LOPU sessions (Pierson et al., 2004; Gibbons et al., 2007; Teixeira et al., 2011; Sanchez et al., 2014). Other authors reported the presence of small adhesions in around $30 \%$ of sheep after repeated LOPU over a 10 week period (McEvoy et al., 2006). In our experience, although some adhesion or fibrosis may be eventually 
found in few animals after several follicular aspiration sessions, it does not represent a relevant limitation.

\section{Manipulation of follicular growth}

In order to recover a high number of good quality COCs per LOPU session, the ovarian status must be synchronized and stimulated using gonadotropins. For synchronization, different protocols have been used in both sheep and goats and most of them are based in estrus synchronization treatments, mainly with progesterone or progestogen-containing intravaginal devices (e.g. CIDR; sponges). Usually, the devices are applied during 9-11 days together with a luteolytic dose of prostaglandin (PG) F2alpha or an analog at the time of gonadotropin treatment start. After synchronization, several treatments have been proposed for follicular stimulation prior to LOPU, being the most popular the multiple FSH injection regime and the socalled oneshot regime, consisting of one dose of FSH plus equine chorionic gonadotropin (eCG) described by Baldassarre and colleagues (Baldassarre et al., 2002).

Different studies in sheep and goats have shown that in vitro matured (IVM) oocytes obtained by follicular aspiration have lower oocyte competence when compared to those maturated in vivo (Cognie et al., 2003), suggesting unsuitable IVM conditions, but also low quality of the oocyte population obtained by aspiration. Oocyte competence is affected by the follicular size from which the COC is collected (Crozet et al., 1995) and also by follicular wave dynamics, growth status and dominance further studied in cattle (Adams et al., 2008). The ideal method should ensure a homogeneous pool of medium $(\sim 4 \mathrm{~mm})$ and young follicles in growing or static phase containing healthy oocytes, and never in late atresia. With this in mind, recently we have tested new approaches for follicular control before LOPU in sheep. Previously, we had described the Day 0 protocol for stimulation of the first follicular wave, for superovulation in conventional embryo production or MOET programs (Menchaca et al., 2002, 2007, 2009, 2010). This protocol synchronizes the ovulation (defined as Day 0 of the cycle) to induce the emergence of the first follicular wave 72 to $84 \mathrm{~h}$ after progesterone device removal and eCG administration. In Day 0 Protocol for MOET programs, FSH is administered in several doses (e.g. 6 or 8 doses) twice a day with the first dose given $84 \mathrm{~h}$ after device removal (i.e. soon after ovulation), coinciding with follicular recruitment of wave 1 . Recently, we have validated this protocol associated to LOPU. In this case, a single dose of 80-100 mg of pFSH reconstituted in sodium hyaluronic acid (MAP-5, Vetoquinol) for slow releasing was given on Day 0 (i.e. $84 \mathrm{~h}$ after device removal). LOPU was performed $72 \mathrm{~h}$ after FSH dose (i.e. on Day 3). Preliminary data show higher number of aspirated follicles and collected oocytes using the Day 0 protocol with pFSH in MAP-5 when compared with those obtained from no FSH treated ewes (Cuadro et al.; IRAUy, Montevideo, Uruguay; unpublished results). Interestingly, the effect of pFSH in MAP-5 used in Day 0 protocol was potentiated when an intravaginal progesterone releasing device (DICO $0.3 \mathrm{~g}$ ) was inserted from Day 0 (i.e. at FSH injection) until the day of LOPU. Follicular development in the presence of high progesterone levels significantly enhanced COCs quality, oocyte fertilization rate, and embryo production (Cuadro et al.; IRAUy, Montevideo, Uruguay; unpublished results). The addition of an intravaginal progesterone releasing device during the follicular development seems to be recommended during the stimulation of wave 1 .

\section{In vitro maturation}

The procedure for in vitro embryo production in sheep and goats has been extensively studied and is further described in previous reviews (Cognie et al., 2004; Paramio, 2010; Souza-Fabjan et al., 2014; Paramio and Izquierdo, 2014, 2016). For that reason, this document describes only the main features and weaknesses of this process.

Regarding IVM, probably most of the effort has been focused on improving the conditions of in vitro culture media. In that sense, a variety of media system have been studied, proposed and adopted. In general, most laboratories use TCM199 supplemented with different components for this stage. This medium can be supplemented with gonadotropins as LH, FSH or a combination of both since it increases the number of oocytes reaching MII and improves the rate of viable embryos (Moor and Trounson, 1977). In addition, cysteamine supplementation acts as a glutathione precursor, improving oocyte maturation clearly demonstrated in several species included sheep and goats (de Matos et al., 2002; Cognie et al., 2003). Estradiol is another proposed component to be included into maturation medium since it is present in the preovulatory follicular fluid. However, its effect is controversial (Guler et al., 2000) and seems to be depending on the presence/absence of other components. Although in lower concentration than follicular fluid, estradiol is present in another component normally added to the IVM medium, the estrus sheep/goat serum. Estrus sheep/goat serum is routinely used in our laboratory, although its variability is well known, containing each batch different levels of metabolites, growth factors, hormones, proteins, lipids, etc., making it difficult to standardize culture conditions. In addition, as a biological component, serum represents a sanitary risk that may be relevant for commercial application of IVEP. On the other hand, chemically defined IVM media have greater repeatability and are safer from a sanitary point of view, deserving future investigation, validation and adoption. A deeper knowledge about the role of follicular environment in oocyte maturation, the function of its components and the interaction between the oocyte and cumulus cells, will help to improve IVEP in the future.

Probably, there are as many variations in embryo culture media as IVEP labs in the world. In our laboratory, maturation medium is composed of TCM199 supplemented with estrus sheep serum, FSH, LH, cysteamine and antibiotics. Excellent and good quality 
oocytes are incubated in groups of $25-30$ per $100 \mu \mathrm{l}$ drops of IVM medium under mineral oil for $24 \mathrm{~h}$ at $39^{\circ} \mathrm{C}$ in a humidified atmosphere containing $5 \% \mathrm{CO}_{2}$. Normally, more than $90 \%$ of metaphase II oocytes is expected to obtain under these conditions.

\section{In vitro fertilization (IVF)}

There are different strategies to prepare ovine/caprine semen for IVF, similar to those used in cattle. Motile spermatozoa are obtained by Percoll gradient or swim-up method and added to COCs in fertilization medium after IVM. Sperm capacitation is normally achieved with the use of heparin added to the fertilization medium and during semen preparation before IVF (e.g. during swim-up). In addition, ionomycin has been tested to improve fertilization rates in goats with good results (Urdaneta et al., 2004).

Some laboratories remove cumulus cells at the end of IVM just before IVF (Cognie et al., 2004). The effect of cumulus cells during IVF has been studied in sheep (Menchaca et al., 2012) and goats (Souza et al., 2013). We found a positive effect of cumulus cells during IVF, with greater cleavage rate $(85$ vs. $77 \%$; $\mathrm{P}<0.05)$ and developmental rates (37 vs. $17 \%$; $\mathrm{P}<0.05)$ when cumulus cells were maintained during IVF than when removed prior to IVF, respectively $(\mathrm{P}<0.05)$. Similar improvement has been reported in goats with greater blastocysts yield (48 vs. 37\%; $\mathrm{P}<0.05$ ) when cumulus cells were maintained during IVF (Souza et al., 2013). After testing several protocols and components, in our laboratory we use synthetic oviduct fluid (SOF) supplemented with heparin, hypotaurine and estrus sheep serum, both for IVF medium and sperm preparation medium. Spermatozoa are capacitated by swim-up with an incubation time of $15 \mathrm{~min}$, and an insemination dose of $1 \times 10^{6}$ total sperm per drop of 100 $\mu \mathrm{l}$ SOF under mineral oil containing 20-30 oocytes. Oocytes and sperm are co-incubated for 18 to $22 \mathrm{~h}$ at $39^{\circ} \mathrm{C}$ in a humidified atmosphere containing $5 \% \mathrm{CO}_{2}$ Working under these conditions with frozen semen, processing more than 6,000 COCs during the last year, the cleavage rate at $48 \mathrm{~h}$ from insemination ranged from 80 to $90 \%$ in more than $80 \%$ of the IVP sessions.

\section{In vitro culture (IVC)}

Four events are crucial during IVEP: cleavage, embryonic genome activation (8-16 cells), morula compaction, and blastocyst formation accompanied by the formation of the inner cell mass and trophectoderm (Lonergan et al., 2003). These events are negatively affected by inadequate culture conditions and several strategies have been designed to mimic the female tract in the lab, with approaches that include in vivo culture of zygotes on the oviduct of temporary recipients, in vitro co-culture with somatic cell support, and semidefined media to suit embryo requirements (Paramio and Izquierdo, 2016).

Different culture media have been tested in small ruminant's embryos; however, SOF medium first described in the 1970s (Tervit et al., 1972) is the most used among laboratories to culture sheep embryos and has been adapted to numerous species including cattle, pigs and goats. In our experience, changing or refreshing IVC medium on Day 3 after insemination improved blastocyst yield on Day 6 from 33.6\% $(78 / 232)$ to $42.0 \%(102 / 243 ; \mathrm{P}<0.05)$, probably by providing fresh nutrients and removing toxic metabolites (Vilariño et al., 2012). Usually embryos are maintained in groups during IVC since this improves blastocysts yields than those single cultured (Gardner and Lane, 1993). In our laboratory, IVC is performed in groups of 20-30 embryos in $100 \mu \mathrm{l}$ drops using SOF under mineral oil and supplemented with BSA, essential and nonessential amino acids and antibiotics, at $39^{\circ} \mathrm{C}$ in $5 \% \mathrm{O}_{2}, 5 \% \mathrm{CO}_{2}$ and $90 \% \mathrm{~N}_{2}$. Expected blastocyst rate under these conditions is around 30 to $40 \%$ (number of blastocysts on Day 6 from COCs in IVF).

\section{Transfer of IVP embryos}

Embryo transfer outcomes could be affected by the embryo quality and by intrinsic and extrinsic factors from the recipient (breed, age, reproductive status, nutrition and health). Regarding embryo quality, survival rate progressively increases with the stage of embryo development for in vivo derived embryos (Bari et al., 2003). Although information for IVP embryos in small ruminants is scarce, the convenience of embryo transfer in blastocyst stages instead of morulae is largely accepted. Regarding the maternal component, the recipient female should provide a competent luteal function with sufficient progesterone production as well as a suitable uterine environment for embryo development and placentation. The luteal activity is determined, at least in part, by the treatment applied to synchronize the ovulation. In this sense, traditional hormonal protocols consist of progesterone or progestogen-based treatment usually administrated with intravaginal devices for 10 to 14 days (goats and sheep, respectively) and associated to eCG administration i.m. at device removal, with a luteolytic dose of PGF2alpha in goats. Alternatively, short-term protocols with progestogen treatment during 6-7 days have been used in sheep with similar outcomes than traditional treatments (Menchaca et al.; IRAUy, Montevideo, Uruguay; unpublished data). Using this protocol for estrus synchronization, around $90 \%$ of estrus and ovulation occurs in average 30 and $60 \mathrm{~h}$ from device removal, respectively (Menchaca and Rubianes, 2004). In our routine in sheep, the intravaginal device is inserted on Day 0 and removed in the evening of Day 6 plus PGF2alpha and 300 IU eCG, and marker vasectomized males are introduced into the flock from 24 to $48 \mathrm{~h}$ after device removal. Fixed time embryo transfer (FTET) is performed on Day 15 (i.e. 8.5 days after device removal and around 7 days after the onset of estrus) to transfer day 6 blastocysts (i.e. 7 days after follicular aspiration). FTET is carried out only in painted females (i.e. those that were mounted by the males) and the embryos are transferred into the uterine horn ipsilateral to the corpus luteum, assessed by laparoscopy. Expected pregnancy rate is approximately 
$50-60 \%$ when FTET is conducted with fresh embryos. Alternatively, FTET may be performed into the oviduct on Day 10, i.e. 3.5 days after device removal and one day after in vitro insemination and ovulation.

The recommended number of IVP embryos to be transferred per recipient is 1 or 2 embryos when FTET is performed with blastocysts, depending on the species, breed and productive system. In our experience, when transferring two IVP embryos around $30 \%$ of pregnant ewes get pregnant with twins. In a recent trial in which FTET was conducted with 240 IVP embryos in Merino recipients (a breed having around 1.1 of prolificity), pregnancy rate was about $10 \%$ greater when two embryos were transferred $(\mathrm{P}<0.05)$. However, birth weight was $20 \%$ lower in twins $(\mathrm{P}<0.05)$ and lamb survival rate after birth was much greater with single pregnancies (about 30\%; $\mathrm{P}<0.05$ ) (Menchaca et al.; IRAUy, Montevideo, Uruguay; unpublished results). For this reason, under typical rangeland conditions as in Uruguay and Argentina, the objective should be to achieve one born lamb per ewe, which ensure a suitable birth weight and lamb survival rate.

\section{Are we ready to skip the fresh embryo transfer?}

One of the greatest difficulties of in vitro embryo technology in livestock is the low cryotolerance of the produced embryos in comparison to in vivo derived (IVD) embryos. For this reason, usually IVEP programs are conducted with fresh embryos, which in large-scale programs with embryos produced every week during long periods of time, require a large and continuous number of ready-to-use recipients. In this context, embryo cryopreservation deserve to be considered.

Conventional slow freezing methods were developed for IVD embryos, and in fact, they are widely applied in sheep and goats in commercial MOET programs. In general, this conventional method results in very low survival rates when applied to IVP embryos (Massip, 2001). In vitro produced embryos have some intrinsic differences with IVD embryos that negatively affect cryotolerance, associated with excessive accumulation of lipids, altered metabolism, changes in structural and physic characteristic, among others (Seidel, 2006). For this reason, vitrification methods have been further studied in parallel with the development of IVEP technology in different species, applied in livestock and endangered species, but also demanded in human assisted reproduction for oocyte and embryo cryopreservation. Several studies have been conducted to test different vitrification methods for small ruminants embryos (Traldi et al., 1999; Dattena et al., 2000; Baril et al., 2001; Martínez et al., 2006; Gibbons et al., 2011; Morato et al., 2011). In general, the effectiveness of vitrification technique depends on several factors as the stage of embryo development, embryo origin (in vivo or in vitro), volume and cooling rate, cryoprotectant media, and the species, among others (Arav, 2014).

During the last years, novel concepts related to vitrification have supported the development of minimum volume methods, reducing the volume of cryoprotectants and increasing the cooling and warming rates (Yavin and Arav, 2007). We have conducted a series of experiments with ovine embryos to improve survival rate after cryopreservation by using vitrification with minimum volume methods (Cryotop and Spatula MVD). These two vitrification methods have been previously reported for human (Kuwayama, 2007) and mice embryos (Tsang and Chow, 2009), respectively. In a recent study, we found that ovine IVP embryos vitrified with Cryotop and Spatula MVD showed acceptable in vitro survival and development rate (dos Santos Neto et al., 2015). In a more recent study (dos Santos Neto et al.; IRAUy, Montevideo, Uruguay; unpublished results), we transferred 437 embryos to compare in vivo vs. in vitro sheep embryos subjected to vitrification by Cryotop or Spatula MVD methods, or to conventional freezing. As expected, regardless the cryopreservation method, pregnancy establishment was greater with IVD than with IVP embryos (pregnancy rate $68.8 v s .22 .3 \%, \mathrm{P}<0.05$ ). Interestingly, recipient females receiving IVD or IVP embryos resulted in greater pregnancy rate $(\mathrm{P}<0.05)$ when vitrification was performed by Cryotop method (77.8 and 55.1\%) than Spatula MVD (59.3 and $18.4 \%$ ) or conventional freezing methods (64.9 and $11.1 \%$, respectively). Thus, in these conditions, the Cryotop method reached $>50 \%$ of pregnant females transferred with IVP embryos. Pregnancy losses from 30 days of gestation to delivery tended to be greater for IVP than IVD embryos (14.9 vs. $5.8 \% ; \mathrm{P}=0.08)$, with no effect of the cryopreservation method (dos Santos Neto et al.; IRAUy, Montevideo, Uruguay; unpublished data). Therefore, minimum volume vitrification methods seems to be interesting for future implementation in IVEP programs in sheep. However, some refinements are still necessary in order to have an easy and robust method to be applied on the field, and thus, finally take advantage of the benefits of cryopreservation.

\section{Concluding remarks}

The cumulative work achieved during several years by the contribution of many researchers, briefly summarized in this review, have transformed the IVEP in a supporting tool for livestock, genetic engineering and research. However, some limitations needs to be solved in order to improve the outcomes and simplify the technique. Oocyte competence is variable and should be improved, mainly by controlling follicular wave dynamics to offer more healthy oocytes for LOPU. In vitro COCs maturation, fertilization and embryo culture is a well-standardized procedure, but still requires some fine-tuning since most of the embryos die during the process before hatching. Embryo transfer and recipients management may be improved by FTET and standardized protocols for ovarian synchronization, as proposed in this review. In addition, fresh embryo transfer is a limiting factor for international exchange of genetic material and requires continuous availability of recipients. In this sense, new minimum volume methods for vitrification seems to be 
attractive allowing acceptable pregnancy rates in our system. In summary, although some challenges are still present in IVEP in sheep and goats, substantial improvements have been achieved during the last years and this technology is available to be applied on the field.

\section{References}

Adams GP, Jaiswal R, Singh J, Malhi P. 2008. Progress in understanding ovarian follicular dynamics in cattle. Theriogenology, 69:72-80.

Arav A. 2014. Cryopreservation of oocytes and embryos. Theriogenology, 81:96-102.

Baldassarre H, de Matos DG, Furnus CC, Castro TE, Cabrera Fischer EI. 1994. Technique for efficient recovery of sheep oocytes by laparoscopic folliculocentesis. Anim Reprod Sci, 35:145-150.

Baldassarre H, Wang B, Kafidi N, Keefer C, Lazaris A, Karatzas C. 2002. Advances in the production and propagation of transgenic goats using laparoscopic ovum pick-up and in vitro embryo production technologies. Theriogenology, 57:275-284.

Baldassarre $\mathbf{H}$, Keefer $\mathbf{C}$, Wang $\mathbf{B}$, Lazaris A, Karatzas CN. 2003a. Nuclear transfer in goats using in vitro matured oocytes recovered by laparoscopic ovum pick-up. Cloning Stem Cells, 5:279-285.

Baldassarre H, Wang B, Kafidi N, Gauthier M, Neveu N, Lapointe J, Sneek L, Leduc M, Duguay F, Zhou JF, Lazaris A, Karatzas CN. 2003b. Production of transgenic goats by pronuclear microinjection of in vitro produced zygotes derived from oocytes recovered by laparoscopy. Theriogenology, 59:831-839.

Baldassarre H, Karatzas CN. 2004. Advanced assisted reproduction technologies (ART) in goats. Anim Reprod Sci, 82/83:255-266.

Bari F, Khalid M, Haresign W, Murray A, Merrell B. 2003. Factors affecting the survival of sheep embryos after transfer within a MOET program. Theriogenology, 59:1265-1275.

Baril G, Traldi AL, Cognie Y, Leboeuf B, Beckers JF, Mermillod P. 2001. Successful direct transfer of vitrified sheep embryos. Theriogenology, 56:299-305.

Cognie Y, Baril G, Poulin N, Mermillod P. 2003. Current status of embryo technologies in sheep and goat. Theriogenology, 59:171-188.

Cognie Y, Poulin N, Locatelli Y, Mermillod P. 2004. State-of-the-art production, conservation and transfer of in-vitro-produced embryos in small ruminants. Reprod Fertil Dev, 16:437-445.

Crozet N, Ahmed-Ali M, Dubos MP. 1995 Developmental competence of goat oocytes from follicles of different size categories following maturation, fertilization and culture in vitro. $J$ Reprod Fertil, 103:293-298.

Dattena M, Ptak G, Loi P, Cappai P. 2000. Survival and viability of vitrified in vitro and in vivo produced ovine blastocysts. Theriogenology, 53:1511-1519.

de Matos DG, Gasparrini B, Pasqualini SR, Thompson JG. 2002. Effect of glutathione synthesis stimulation during in vitro maturation of ovine oocytes on embryo development and intracellular peroxide content. Theriogenology, 57:1443-1451. dos Santos Neto PC, Vilarino M, Barrera N, Cuadro F, Crispo M, Menchaca A. 2015. Cryotolerance of Day 2 or Day 6 in vitro produced ovine embryos after vitrification by Cryotop or Spatula methods. Cryobiology, 70:17-22.

Gardner DK, Lane M. 1993. Embryo culture systems. In: Gardner DK, Trounson AO( Ed.). Handbook of In Vitro Fertilization. Boca Raton, FL: CRC Press. pp. 84105.

Gibbons A, Pereyra Bonnet F, Cueto MI, Catala M, Salamone DF, Gonzalez-Bulnes A. 2007. Procedure for maximizing oocyte harvest for in vitro embryo production in small ruminants. Reprod Domest Anim, 42:423-426.

Gibbons A, Cueto MI, Pereyra Bonnet F. 2011. A simple vitrification technique for sheep and goat embryo cryopreservation. Small Rumin Res, 95:61-64.

Guler A, Poulin N, Mermillod P, Terqui M, Cognie Y. 2000. Effect of growth factors, EGF and IGF-I, and estradiol on in vitro maturation of sheep oocytes. Theriogenology, 54:209-218.

Kuwayama M. 2007. Highly efficient vitrification for cryopreservation of human oocytes and embryos: the Cryotop method. Theriogenology, 67:73-80.

Lonergan P, Rizos D, Gutierrez-Adan A, Fair T, Boland MP. 2003. Oocyte and embryo quality: effect of origin, culture conditions and gene expression patterns. Reprod Domest Anim, 38:259-267.

Martínez AG, Valcárcel A, Furnus CC, de Matos DG, Iorio G, de las Heras MA. 2006. Cryopreservation of in vitro-produced ovine embryos. Small Rumin Res, 63:288-296.

Massip A. 2001. Cryopreservation of embryos of farm animals. Reprod Domest Anim, 36:49-55.

McEvoy TG, Alink FM, Moreira VC, Watt RG, Powell KA. 2006. Embryo technologies and animal health - consequences for the animal following ovum pick-up, in vitro embryo production and somatic cell nuclear transfer. Theriogenology, 65:926-942.

Menchaca A, Pinczak A, Rubianes E. 2002. Follicular recruitment and ovulatory response to $\mathrm{FSH}$ treatment initiated on Day 0 or Day 3 postovulation in goats. Theriogenology, 58:1713-1721.

Menchaca A, Rubianes E. 2004. New treatments associated with timed artificial insemination in small ruminants. Reprod Fertil Dev, 16:403-413.

Menchaca A, Vilarino M, Crispo M, Pinczak A, Rubianes E. 2007. Day 0 protocol: superstimulatory treatment initiated in the absence of a large follicle improves ovarian response and embryo yield in goats. Theriogenology, 68:1111-1117.

Menchaca A, Vilarino M, Pinczak A, Kmaid S, Saldana JM. 2009. Progesterone treatment, FSH plus eCG, GnRH administration, and Day 0 Protocol for MOET programs in sheep. Theriogenology, 72:477-483. Menchaca A, Vilarino M, Crispo M, de Castro T, Rubianes E. 2010. New approaches to superovulation and embryo transfer in small ruminants. Reprod Fertil Dev, 22:113-118.

Menchaca A, Vilariño $M$, dos Santos Neto PC, Wijma R, Crispo M. 2012. Cumulus cells are involved in oocyte maturation and fertilization in vitro produced 
ovine embryos. Reprod Fertil Dev, 47(4).

Menchaca A, Anegon I, Whitelaw CB, Baldassarre H, Crispo M. 2016. New insights and current tools for genetically engineered (GE) sheep and goats. Theriogenology, 86:160-169.

Moor RM, Trounson AO. 1977. Hormonal and follicular factors affecting maturation of sheep oocytes in vitro and their subsequent developmental capacity. $J$ Reprod Fertil, 49:101-109.

Morato R, Romaguera R, Izquierdo D, Paramio MT, Mogas T. 2011. Vitrification of in vitro produced goat blastocysts: effects of oocyte donor age and development stage. Cryobiology, 63:240-244.

Paramio MT. 2010. In vivo and in vitro embryo production in goats. Small Rumin Res, 89:144-148.

Paramio MT, Izquierdo D. 2014. Current status of in vitro embryo production in sheep and goats. Reprod Domest Anim, 49(suppl. 4):37-48.

Paramio MT, Izquierdo D. 2016. Recent advances in in vitro embryo production in small ruminants. Theriogenology, 86:152-159.

Pierson J, Wang B, Neveu N, Sneek L, Côté F, Karatzas CN, Baldassarre H. 2004. Effects of repetition, interval between treatments and season on the results from laparoscopic ovum pick-up in goats Reprod Fertil Dev, 16:795-799.

Sanchez DJD, Melo CHS, Souza-Fabjan JMG, Sousa FC, Rocha AA, Campelo IS, Teixeira DIA, Pereira AF, Melo LM, Freitas VJF. 2014. Repeated hormonal treatment and laparoscopic ovum pick-up followed by in vitro embryo production in goats raised in the tropics Livest Sci, 165:217-222.

Seidel GE Jr. 2006. Modifying oocytes and embryos to improve their cryopreservation. Theriogenology, 65:228-235

Souza JM, Duffard N, Bertoldo MJ, Locatelli Y, Corbin E, Fatet A, Freitas VJ, Mermillod P. 2013. Influence of heparin or the presence of cumulus cells during fertilization on the in vitro production of goat embryos. Anim Reprod Sci, 138:82-89.

Souza-Fabjan JM, Panneau B, Duffard N, Locatelli Y, Figueiredo JR, Freitas VJ, Mermillod P. 2014. In vitro production of small ruminant embryos: late improvements and further research. Theriogenology, 81:1149-1162.

Teixeira PP, Padilha LC, Oliveira ME, Motheo TF, Silva AS, Barros FF, Coutinho LN, Flores FN, Lopes MC, Bandarra MB, Silva MA, Vasconcelos RO, Rodrigues LF, Vicente WR. 2011. Laparoscopic ovum collection in sheep: gross and microscopic evaluation of the ovary and influence on ooctye production. Anim Reprod Sci, 127:169-175.

Tervit HR, Whittingham DG, Rowson LE. 1972. Successful culture in vitro of sheep and cattle ova. $J$ Reprod Fertil, 30:493-497.

Tervit HR, Smith JF, McGowan LT, Wells RW, Parr J. 1992. Laparoscopic recovery of oocytes from sheep. Proc Aust Soc Reprod Biol, 24:26.

Traldi AS, Leboeuf B, Cognié Y, Poulin N, Mermillod P. 1999. Comparative results of in vitro and in vivo survival of vitrified in vitro produced goat and sheep embryos. Theriogenology, 51:175. (abstract).

Tsang WH, Chow KL. 2009. Mouse embryo cryopreservation utilizing a novel high-capacity vitrification spatula. Biotechniques, 46:550-552.

Urdaneta A, Jimenez AR, Paramio MT, Izquierdo D. 2004. Cysteamine, glutathione and ionomycin treatments improve in vitro fertilization of prepubertal goat oocytes. Zygote, 12:277-284.

Vilariño M, Crispo M, dos Santos-Neto PC, Wijma R, Menchaca A. 2012. The effect of culture medium changes on in vitro production of sheep embryos. Reprod Domest Anim, 47:1806.

Yavin S, Arav A. 2007. Measurement of essential physical properties of vitrification solutions. Theriogenology, 67:81-89. 